\title{
Programa de Educação pelo Trabalho e para a Saúde (PET - Saúde) \\ Interprofissionalidade: Aproximações e distanciamentos com as definições da
}

\section{Educação Interprofissional}

\author{
Education through Work and Health Program (PET - Saúde) Interprofessionality: Approaches and \\ distances with the definitions of Interprofessional Education
}

Programa Educación por el Trabajo y la Salud (PET - Saúde) Interprofesionalidad: Aproximaciones y alejamientos con las definiciones de la Educación Interprofesional

Francisca Juliana Rocha Torres ORCID: https://orcid.org/0000-0001-9557-6157 Universidade Estadual Vale do Acaraú, Brasil E-mail: francisca.jrt@gmail.com

Ana Clara de Souza Correa ORCID: https://orcid.org/0000-0001-9395-4732 Universidade Federal do Ceará, Brasil E-mail: aclarasouza98@gmail.com

Cibelly Aliny Siqueira Lima Freitas ORCID: https://orcid.org/0000-0002-0585-5345

Universidade Estadual Vale do Acaraú, Brasil E-mail:cibellyaliny@gmail.com

Ricardo Lima dos Santos

ORCID: https://orcid.org/0000-0001-8481-1414 Universidade Estadual Vale do Acaraú, Brasil E-mail: richlima@ hotmail.com

Antonia Rochelly Marques Rodrigues ORCID: https://orcid.org/0000-0001-6249-0488 Centro de Saúde da Família Herbert de Sousa, Brasil E-mail: rochelly_rodrigues@hotmail.com

Maria Socorro de Araújo Dias

ORCID: https://orcid.org/0000-0002-7813-547X Universidade Estadual Vale do Acaraú, Brasil E-mail: socorroad@gmail.com

\begin{abstract}
Resumo
O Programa de Educação pelo Trabalho e para a Saúde (PET-Saúde) trouxe na sua nona edição a temática em torno da Educação Interprofissional (EIP), visando a qualificação na formação de estudantes e profissionais, a partir de ações e discussões que articulem o ensino-serviço-comunidade. Dessa forma, este ensaio tem como objetivo descrever a percepção de um grupo tutorial do PET-Saúde/Interprofissionalidade sobre os distanciamentos e as aproximações das ações do programa com relação aos elementos essenciais da Educação Interprofissional. Trata-se de um estudo descritivo do tipo relato de experiência, sistematizado por integrantes do PET-Saúde/Interprofissionalidade Sobral, Ceará. As percepções dos integrantes foram resgatadas por meio da aplicação de um formulário criado no Google forms durante o mês de março de 2020. Assim, pode-se observar que os principais avanços envolvem a constituição dos grupos, pois, contemplam estudantes e profissionais de diferentes áreas, onde os participantes aprendem juntos e dialogam com outros saberes. Dentre as principais dificuldades ou distanciamentos estão a necessidade de uma maior integração entre os grupos tutoriais. Outro obstáculo posto, foi o desafio de trabalhar a interprofissionalidade no cenário de prática dos profissionais e docentes, pois, muitos não conhecem o conceito e estão acostumados a desenvolver suas atividades de forma uniprofissional. Conclui-se reforçando o Programa PET-Saúde como um espaço privilegiado para a construção de saber e diálogo entre as profissões, sendo importante buscar soluções para os desafios das vivências, reconhecendo a importância da atuação interprofissional e a qualificação da assistência à população em defesa do Sistema Único de Saúde (SUS).

Palavras-chave: Educação interprofissional; Práticas interdisciplinares; Relações interprofissionais; Educação em saúde; Assistência integral à saúde.
\end{abstract}




\begin{abstract}
The Education through Work and Health Program (PET-Saúde) brought in its ninth edition the theme around Interprofessional Education (EIP), aiming at the qualification in the training of students and professionals, based on actions and discussions that articulate the teaching-service-community. Thus, this essay objective is to describe the perception of a PET-Saúde/ Interprofessional tutorial group on the distances and approximations of the program's actions in relation to the essential elements of Interprofessional Education. This is a descriptive study of the experience report type, systematized by members of PET - Health/Interprofessional Sobral, Ceará. The members' perceptions were recovered through the application of a form created in Google Forms during the month of March 2020. That said, it can be seen that the main advances involve the constitution of groups, as they contemplate students and professionals from different areas, where participants learn together and dialogue with other knowledge. Among the main difficulties or distances are the need for greater integration between tutorial groups. Another obstacle, was the challenge of working interprofessionality in the practice scenario of professionals and teachers, as many do not know the concept and are used to developing their activities in a uniprofessional way. It concludes by reinforcing the PET Health/Interprofessional as a privileged space for the construction of knowledge and dialogue between professions, it is important to seek solutions to the challenges of the experiences, recognizing the importance of interprofessional performance and the qualification of assistance to the population in defense of the Unified Health System.
\end{abstract}

Keywords: Interprofessional education; Interdisciplinary placement; Interprofessional relations; Health education; Comprehensive health care.

\title{
Resumen
}

El Programa de Educación para el Trabajo y para la Salud (PET-Salud) ha traído en su novena edición la temática entorno de la Educación Interprofesional (EIP), con el fin a la cualificación en la formación de estudiantes y profesionales, a partir de acciones y discusiones que articulen la enseñanza-servicio-comunidad. De esa forma, este ensayo tiene como objetivo describir la percepción de un grupo tutorial del PET-Salud/Interprofesionalidad acerca de los alejamientos y aproximaciones de las acciones del programa en relación a los elementos esenciales de la Educación Interprofesional. Se trata de un estudio descriptivo del tipo relato de experiencia, sistematizado por los integrantes del PET-Salud/Interprofesionalidad Sobral, Ceará. Las percepciones de los integrantes fueron rescatadas por medio de la aplicación de un formulario creado en el Google Forms durante el mes de marzo de 2020. Dicho esto, se puede observar que los principales avances envuelven la constitución de los grupos, pues, contemplan estudiantes y profesionales de diferentes áreas, donde los participantes aprenden juntos y dialogan con otros saberes. Entre las principales dificultades o alejamientos están la necesidad de una mayor integración entre los grupos tutoriales. Otro entrave puesto, fue el desafío de trabajar la interprofesionalidad en el escenario de práctica de los profesionales y docentes, pues, muchos no conocen el concepto y están acostumbrados a desarrollar sus actividades de forma uniprofesional. Se concluye reforzando el Programa PET-Salud como un espacio privilegiado para la construcción del saber y diálogo entre las profesiones, siendo importante buscar soluciones para los desafíos de las vivencias, reconociendo la importancia de la actuación interprofesional y la cualificación de la asistencia a la población en defensa del Sistema Único de Salud (SUS). Palabras clave: Educación interprofesional; Prácticas interdisciplinarias; Relaciones interprofesionales; Educación en salud; Atención integral de salud.

\section{Introdução}

No Brasil, as discussões e iniciativas sobre as práticas interprofissionais e colaborativas, no campo do trabalho, acompanham a criação e a implementação do Sistema Único de Saúde (SUS). Contudo, as discussões na área da formação dos profissionais da saúde são, relativamente, recentes (Freire Filho, Silva, Costa \& Forster, 2019). Ainda persiste no país, uma formação voltada para o uniprofissionalismo, o que fragmenta o atendimento e o cuidado ao paciente, divergindo do princípio de integralidade, estabelecido pelo SUS (Costa, Freire Filho, Brandão \& Silva, 2018). Por isso, é fundamental integrar e discutir a interprofissionalidade não só no âmbito profissional, mas também na universidade, para que os estudantes já saiam das instituições de ensino aptos para o mercado e as demandas do sistema.

Nesse sentido, a Educação Interprofissional (EIP) surge com o objetivo de estimular a interação entre os profissionais, sendo caracterizada pela junção de duas ou mais profissões que aprendem entre si, de forma interativa, para juntos melhorar a qualidade da atenção à saúde, com base nas necessidades dos usuários/comunidade. Desse modo, a EIP visa a construção de um espaço de aprendizagem que favoreça o diálogo entre as profissões, trabalhando para o desenvolvimento de atitudes, habilidades e competências que estimulem os profissionais ao trabalho em equipe, com base na colaboração interprofissional (Reeves, 2016). 
Além disso, a EIP propõe uma transformação no processo de formação em saúde, norteados pela importância do trabalho em equipe, o reconhecimento e respeito às especificidades de cada profissão para juntos atuarem na tomada de decisões e na resolução dos problemas, preparando futuros profissionais que sejam aptos ao trabalho interprofissional. Dessa forma, a EIP mostra-se relevante, pois, busca estratégias e atitudes que contemple uma formação voltada a aprendizagem compartilhada e o uso de metodologias, ofertando aos estudantes competências para a atuação nas esferas de promoção, prevenção e recuperação da saúde, contribuindo na construção de um novo perfil profissional que atenda as necessidades dos pacientes/usuários, com base nos princípios do SUS e buscando a qualificação da assistência (Batista, 2012; Lamers \& Toassi, 2018).

A partir disso, algumas iniciativas foram lançadas em prol de introduzir alguns princípios voltados para o trabalho e a formação em equipe, interprofissional e colaborativa. Um desses projetos pioneiros ocorreu na década de 80 com a implementação do Programa UNI (Uma Nova Iniciativa na Educação dos Profissionais do Setor da Saúde). Apesar de ter sido uma ação mais voltada para os acadêmicos dos cursos de medicina e enfermagem, ele buscou incorporar aos cursos uma abordagem mais interdisciplinar e multidisciplinar (Lima, Ribeiro, Padilha \& Mourthé Júnior, 2018).

Outro grande avanço ocorreu em 2003 com a criação da Secretaria de Gestão do Trabalho e da Educação em Saúde (SGTES), que possibilitou a institucionalização de ações e políticas voltadas para a educação na saúde e da reorientação na formação, o que mais tarde possibilitaria a criação do Programa de Residência Multiprofissional em Saúde, o Pró-Saúde (Programa Nacional de Reorientação da Formação Profissional em Saúde) e o PET-Saúde (Programa de Educação pelo Trabalho para a Saúde), que se assemelham no quesito de desenvolvimento de competências com base na interprofissionalidade (Freire Filho et al., 2019; Toassi, 2017).

Em 2017, esses avanços na área da prática interprofissional e colaborativa foram intensificados após a Organização Pan-Americana da Saúde/Organização Mundial da Saúde (OPAS/OMS), que fomentam e reforçam aos países membros a necessidade de implementar intervenções que visavam ampliar a (EIP). Diante desse e de outros chamados emitidos por essa organização, o Brasil elaborou um conjunto de ações com o intuito de implementar a EIP no país, destacando-se o PETSaúde/Interprofissionalidade (Almeida \& Silva, 2019; Costa, Peduzzi, Freire Filho \& Silva, 2018).

Em 2008, foi instituído a portaria interministerial MS/MEC Nº1.802 o PET-Saúde, que foi criado com o objetivo de promover uma formação qualificada dos profissionais, a partir da integração do ensino-serviço-comunidade. Na sua nona edição, esse projeto, atendendo as demandas da OPAS/OMS teve como temática a Educação Interprofissional na Saúde, que recebeu o nome de PET-Saúde/Interprofissionalidade (Brasil, 2008).

Nessa perspectiva, o PET-Saúde/Interprofissionalidade surge com a ideia de transformar o processo de formação profissional, articulando suas ações para o fortalecimento do ensino-serviço-comunidade. Desse modo, o programa tem como propósito contribuir para a formação dos profissionais, estimulando os estudantes para a prática colaborativa, com base na integralidade do cuidado (Camara, Grosseman \& Pinho, 2015; Pereira et al., 2020).

É a partir desse contexto que o PET-Saúde/Interprofissionalidade de Sobral-CE, com o apoio da Secretaria da Saúde de Sobral, da Escola de Saúde Pública Visconde de Sabóia, Universidade Estadual Vale do Acaraú (UVA) e Universidade Federal do Ceará (UFC), vem desenvolvendo intervenções nos serviços e nas universidades participantes do projeto com o objetivo de estimular discussões entre os profissionais e estudantes acerca da EIP e da Prática Colaborativa (PC), e dessa forma, ajudar na transformação da reorientação na formação em saúde, assim como, na melhoria da qualidade da atenção à saúde.

Assim, o presente estudo tem como objetivo descrever a percepção de um grupo tutorial do PETSaúde/Interprofissionalidade sobre os distanciamentos e as aproximações das ações do programa com relação aos elementos essenciais da Educação Interprofissional. 


\section{Metodologia}

Trata-se de um estudo descritivo do tipo relato de experiência, com abordagem qualitativa e sistematizado por integrantes de um grupo tutorial do PET-Saúde/Interprofissionalidade de Sobral-Ceará. Na pesquisa qualitativa, o pesquisador deve atentar-se à descrição dos fenômenos narrados pelos entrevistados, a fim de obter uma maior clareza dos fatos (Pereira, Shitsuka, Parreira \& Shitsuka, 2018). Dessa forma, foi elaborado um formulário no Google forms com o intuito de registrar as percepções dos integrantes de um grupo tutorial do PET sobre como as ações desenvolvidas no primeiro ano (2019) do Projeto se aproximam ou distanciam dos elementos definidores da EIP.

O formulário foi elaborado e encaminhado para os membros de um grupo tutorial do PET-Saúde/Interprofissionalidade por meio de mídias sociais, durante o mês de março de 2020, com prazo determinado para preenchimento. Foram registradas as percepções de doze integrantes de um dos grupos tutoriais do PET-Saúde, compreendendo estudantes dos Cursos de Psicologia, Medicina, Enfermagem, Odontologia e Educação Física, profissionais do serviço (Enfermeira, Cirurgiã-dentista e Psicólogo) e docentes (Profissional de Educação Física e Enfermeira).

A utilização do formulário eletrônico como meio para coleta dos dados, mostrou-se uma ferramenta bastante eficiente para possibilitar o processo de registro e sistematização de informações.

As percepções sobre as ações desenvolvidas pelo projeto foram analisadas pelos integrantes do PETSaúde/Interprofissionalidade e registrados aqui de forma descritiva, com vistas a evidenciar desafios e potencialidades, os quais foram discutidas à luz da literatura em EIP e PC.

\section{Resultados e Discussão}

As percepções dos integrantes de um grupo tutorial do PET-Saúde/ Interprofissionalidade estão expressos no quadro a seguir, por meio do registro dos aspectos que demonstram aproximação e distanciamento com os elementos essenciais da EIP e da PC.

Quadro 1 - Aproximações e distanciamentos das ações do Projeto PET-Saúde/Interprofissionalidade com as definições da Educação Interprofissional. Sobral, Ceará, Brasil. 2020.

\begin{tabular}{|c|c|}
\hline APROXIMAÇÕES & DISTANCIAMENTOS \\
\hline $\begin{array}{l}\text { - Esforços para inserir no cotidiano da prática os principais } \\
\text { aspectos da EIP; } \\
\text { - Diálogos interprofissionais intragrupais, com vistas a } \\
\text { incentivar a EIP nas graduações; } \\
\text { - Importante abordagem teórica sobre a temática da EIP } \\
\text { subsidiando as discussões do grupo e o processo de } \\
\text { construção do conhecimento para apoiar na formação e no } \\
\text { trabalho em saúde; } \\
\text { - Compartilhamento de saberes dentro do grupo do PET- } \\
\text { saúde, considerando a sua constituição envolvendo } \\
\text { profissionais de categorias diferentes, além da ampliação } \\
\text { desses conhecimentos para outros cenários, como os cursos } \\
\text { de enfermagem, educação física, medicina, psicologia e } \\
\text { odontologia; } \\
\text { - Possibilidade de discussão de um determinado fenômeno a } \\
\text { partir de áreas distintas do conhecimento, evidenciando as } \\
\text { potências de cada Curso; }\end{array}$ & $\begin{array}{l}\text { - Dificuldades no planejamento e desenvolvimento de ações } \\
\text { interprofissionais nos serviços apresentando os conceitos da } \\
\text { EIP; } \\
\text { - Dificuldades de desenvolver um trabalho colaborativo com } \\
\text { os outros grupos do Projeto; } \\
\text { - Lacuna quanto ao desenvolvimento de atividades em sala de } \\
\text { aula e no cotidiano de trabalho que possibilite a } \\
\text { implementação dos conceitos da EIP; } \\
\text { - Reconhecimento de um modelo conservador e hierarquizado } \\
\text { de ensino, que muitas vezes, gera resistência em aderir a } \\
\text { novas formatações (conceitos, conteúdos, metodologias); } \\
\text { - Desenvolvimento de ações com pouco alcance aos demais } \\
\text { estudantes e docentes não envolvidos com o Projeto. } \\
\text { - Desafios na inserção dos aspectos centrais da EIP e da PC } \\
\text { no contexto da Universidade, tanto no que se refere às ligas, } \\
\text { aulas, ambulatórios e, até mesmo no convívio diário da } \\
\text { Universidade; } \\
\text { - Ausência de projetos que integrem os diversos saberes e } \\
\text { estimulem a aprendizagem colaborativa; }\end{array}$ \\
\hline
\end{tabular}




\begin{abstract}
- As atividades realizadas pelo Projeto estimulam a integração interprofissional na medida em que incentivam o diálogo dos professores dos diversos cursos da saúde;

- As atividades do Projeto tem contribuído na concepção e implementação dos currículos dos Cursos envolvidos, trazendo à tona discussões sobre a formação de profissionais sensíveis para o trabalho em equipe e a integralidade do cuidado;

- A configuração dos grupos do Projeto, bem como as ações desenvolvidas têm suscitado a integração ensino-serviço e a incorporação, mesmo que de forma tímida, dos conceitos de EIP e PC;

- As intervenções do PET têm incentivado estudantes/profissionais a importância do trabalho em equipe para a promoção da qualidade da assistência.
\end{abstract}

- Deficiência na integração de docentes dos diversos cursos da área da saúde no trabalho interprofissional, para a construção de uma docência colaborativa, tendo em vista que temos alguns cursos que têm uma maior integração em detrimento de outros.

Fonte: Autores.

No cenário das aproximações, observa-se que o PET-Saúde/Interprofissionalidade começa a trabalhar a EIP desde sua constituição, englobando profissionais/estudantes de diversas áreas do setor saúde, permitindo a interação entre as profissões, fazendo com que os seus participantes reconheçam a importância do papel de cada profissional no processo de cuidado, trabalhando em conjunto na resolução dos problemas, por meio do diálogo e da aprendizagem compartilhada, respeitando a singularidade e o saber de cada profissão, para a melhoria da assistência à saúde (Araújo, Vasconcelos, Pessoa \& Forte, 2017).

Entre outras aproximações estavam as atividades desenvolvidas no projeto que contribuem para repensar na reorientação dos currículos das graduações em saúde, trazendo à tona discussões sobre o processo de formação dos profissionais sensíveis ao trabalho em equipe interprofissional e a integralidade do cuidado. À vista disso, observa-se que o programa visa contribuir para a construção de um novo perfil profissional, pautado na importância de trabalhar em equipe e de contribuir para a consolidação do sistema de saúde do país, considerando os princípios do SUS, principalmente a integralidade.

Nessa perspectiva, destaca um dos objetivos do programa, pois, este busca contribuir para a mudança dos currículos dos cursos das graduações em saúde, de forma que, seja pautado nos princípios da Prática Colaborativa e da Educação Interprofissional, buscando a qualificação da assistência a partir da integração ensino-serviço-comunidade. Desse modo, percebese a necessidade de mudanças nos currículos, tendo em vista que a formação uniprofissional foca somente nas especificidades de cada profissão (Barbosa et al., 2020).

Os integrantes reconheceram o quanto os preceptores têm envidado esforços para discutir com seus pares os elementos essenciais do conceito da EIP para a prática do trabalho, ressaltando a importância do trabalho interprofissional e colaborativo no processo de cuidar em saúde. Outro aspecto importante é a configuração dos grupos tutoriais do Projeto, bem como as ações desenvolvidas que têm suscitado a integração ensino-serviço e a incorporação, mesmo que de forma tímida, dos conceitos de EIP e PC.

Expressões de aproximações das atividades com os elementos da EIP e PC estão as rodas de conversa com os estudantes dos cursos de enfermagem e educação física da Universidade Estadual Vale do Acaraú (UVA); a roda de conversa com os trabalhadores da Estratégia Saúde da Família e a roda de conversa com o Núcleo Docente Estruturante (NDE) dos cursos participantes do PET-Saúde/Interprofissionalidade, entre outras ações desenvolvidas com os profissionais do serviço, docentes e discentes dos cursos envolvidos.

Nesse contexto da Interprofissionalidade, avanços e desafios têm lugar na caminhada. Os distanciamentos envolvem aspectos como uma comunicação pouco efetiva entre os integrantes da equipe de saúde e, a resolução de problemas se dá de forma uniprofissional. 
Observa-se ainda que os currículos apresentam incipiência no que se refere ao desenvolvimento de competências interprofissionais e colaborativas, prevalecendo um modelo de formação uniprofissional, pautado nas especificidades de cada profissão. Isto posto, é possível perceber os obstáculos que impedem mudanças no modelo de ensino em saúde no país, que envolvem desde as estruturas físicas entre os cursos, até a constituição dos currículos que têm suas características definidas, revelando uma fragmentação do ensino e a pouca interação entre os estudantes. Todavia, um dos grandes obstáculos são as barreiras culturais, sendo caracterizada pela formação específica de cada área e com forte inflûencia na identidade profissional, dificultando o desenvolvimento de competências, como a interdependência entre as profissões, integração no trabalho, responsabilidade compartilhada e entre outros (Costa, 2016; Reeves, Xyrichis \& Zwarenstein, 2018).

Isso contribui para que ocorra uma ruptura da construção do conhecimento, fazendo com que um grupo não tome conhecimento das atividades dos demais e nem que haja o compartilhamento de aprendizagem. Por consequência, além da fragmentação do conhecimento e do compartilhamento das experiências, também ocorre uma limitação da imersão dos integrantes do projeto, considerando os objetivos dos eixos os quais estão inseridos.

Apesar de um enriquecimento do conhecimento, das práticas e das ações no âmbito da interprofissionalidade para aqueles indivíduos que estão participando do programa, há um distanciamento em compartilhar o aprendizado de forma mais efetiva com a Comunidade, incluindo os estudantes e profissionais não envolvidos no programa.

Outro aspecto que merece destaque são os desafios no planejamento e desenvolvimento de ações interprofissionais nos serviços, apresentando os conceitos da EIP. O mesmo fato se perpetua no desenvolvimento de atividades em sala de aula e no cotidiano de trabalho que possibilite a implementação dos conceitos da EIP. Muitas vezes, quando ocorre a abordagem da interprofissionalidade e das práticas colaborativas com os profissionais e os estudantes, estas apresentam um caráter mais teórico.

Esse cenário contrapõe-se com os próprios conceitos e pilares envoltos da EIP, que requer uma aprendizagem mais significativa voltada para a interação entre diferentes profissionais em espaço que possibilite não apenas uma aprendizagem teórica, como também a oportunidade de observar e simular esses momentos de interação interprofissional (Batista, 2012).

Outro aspecto que deve ser considerado é a presença de um modelo conservador e hierarquizado de ensino, que muitas vezes, gera resistência em aderir às novas formatações de trabalho, ensino e aprendizagem. Essa realidade também encontra embasamento em outros estudos que apontam a hierarquização como um dos fatores que colaboram para a fragmentação do cuidado e da atenção à saúde (Lima et al., 2018).

Torna-se fundamental o exercício da interprofissionalidade, da prática colaborativa e do trabalho em equipe entre os eixos. Outrossim, é importante ressaltar a dificuldade dos preceptores em aplicarem os aprendizados da EIP e da PC na sua área de atuação e trabalho. Por fim, cabe destacar os desafios na gestão do tempo, uma vez que, muitas vezes, preceptores e estudantes, não conseguem ter governabilidade de suas agendas para dedicarem-se de forma mais efetiva às ações, tanto pela constituição dos currículos, como pela dinâmica de trabalho nos serviços de saúde.

Diante dessa realidade, é importante reafirmar que as intervenções do PET-Saúde/Interprofissionalidade têm incentivado e sensibilizado estudantes e profissionais sobre a importância do trabalho em equipe para a promoção da qualidade da assistência à população.

\section{Considerações Finais}

O PET-Saúde Interprofissionalidade representa um dos grandes avanços para introdução e difusão dos conceitos e das práticas interprofissionais e colaborativas entre os discentes, docentes e trabalhadores da área da saúde no Brasil. Por isso, após um ano do projeto, faz-se necessário analisar, sob a perspectiva de seus integrantes, as aproximações de desafios do projeto. 
O compartilhamento dessa experiência representa uma potente expressão da democratização dos saberes e das vivências em interprofissionalidade. Pode-se observar que os principais avanços envolvem a constituição dos grupos, pois, contemplam estudantes e profissionais de diferentes áreas, onde os participantes aprendem juntos e dialogam com outros saberes.

Dentre os principais distanciamentos está a necessidade de integração entre os grupos tutoriais do Projeto. Outro obstáculo posto, foi o desafio de trabalhar a interprofissionalidade no cenário de prática dos profissionais e docentes, pois, muitos não conhecem o conceito e estão acostumados a desenvolver suas atividades de forma uniprofissional.

No âmbito das universidades é necessário promover uma maior inserção das atividades do grupo PET-Saúde, dos conceitos e das vivências da interprofissionalidade nas atividades da graduação, bem como estabelecer um diálogo mais próximo. Recomenda-se a criação de projetos que integrem os diversos saberes e estimulem o trabalho interprofissional e a aprendizagem colaborativa.

Conclui-se que o Programa PET-Saúde se constitui como um espaço privilegiado para a construção de saberes e diálogo entre as profissões, sendo relevante buscar soluções para os desafios das vivências, reconhecendo a importância da atuação interprofissional e a qualificação da assistência à população em defesa do Sistema Único de Saúde (SUS). Desse modo, destaca a importância de desenvolver mais estudos acerca dos desafios da EIP no Brasil e sua relevância para a saúde pública.

\section{Referências}

Araújo, T. A. M., Vasconcelos, A. C. C. P., Pessoa, T. R. R. F., \& Forte, F. D. S. (2017). Multiprofissionalidade e Interprofissionalidade em uma residência hospitalar: o olhar de residentes e preceptores. Interface (Botucatu), 21(62), 601-613. http://dx.doi.org/10.1590/1807-57622016.0295

Almeida, R. G. S., \& Silva, C. B. G. (2019). A educação interprofissional e os avanços do Brasil. Revista Latino - Americana de Enfermagem, 27. https://doi.org/10.1590/1518-8345.3148-3152

Batista, N. A. (2012). Educação interprofissional em saúde: concepções e práticas. Cad Fnepas. 2:25-8. http://www.fnepas.org.br/artigos_caderno/v2/educacao_interprofissional.pdf

Brasil. Ministério da Saúde. Ministério da Educação. Portaria Interministerial nº1802. Institui o Programa de Educação pelo Trabalho para a Saúde - PET-Saúde. Diário Oficial da União, Brasília, DF, 2008. http://bvsms.saude.gov.br/bvs/saudelegis/gm/2008/pri1802_26_08_2008.html

Barbosa, L. A. S., Torres, F. J. R., Almeida, G. N., Freitas, C. A. S. L., Dias, M. S. A., Santos, R. L., Filho, A. P. P. (2020). Educação Interprofissional e Práticas Colaborativas em Saúde. Ceará: Edições UVA. http://www.uvanet.br/edicoes_uva/gera_xml.php?arquivo=educacao_interprofissional_27112020

Costa, M. V., Peduzzi, M., Freire Filho, J. R. \& Silva, C. B. G., (2018). Educação interprofissional em saúde - Natal: SEDIS-UFRN.

Costa, M. V., Freire Filho, J. R., Brandão, C., \& Silva, J. A. M. (2018). A Educação e o trabalho interprofissional alinhados ao compromisso histórico de fortalecimento e consolidação do Sistema Único de Saúde (SUS). Interface - Comunicação, Saúde, Educação, 22 (Supl. 2). 1507-10. https://doi.org/10.1590/1807-57622018.0636

Costa, M. V. (2016). A educação interprofissional no contexto brasileiro: algumas reflexões. Interface - Comunicação, Saúde, Educação, 20(56), 197-8. https://doi.org/10.1590/1807-57622015.0311

Camara, A. M. C. S., Grosseman, S. \& Pinho, D. L. M. (2015). Educação Interprofissional no Programa PET-Saúde: a percepção de tutores. Interface Comunicação, Saúde, Educação, 19(Supl.1) 817-829. http://dx.doi.org/10.1590/1807-57622014.0940

Freire Filho, J. R., Silva, C. B. G., Costa, M. V., \& Forster, A. C. (2019). Educação Interprofissional nas políticas de reorientação da formação profissional em saúde no Brasil. Saúde em Debate, 43 (spe1), 86-96. https://doi.org/10.1590/0103-11042019s107

Lima, V. V., Ribeiro, E. C. O., Padilha, R. Q., \& Mourthé Júnior, C. A. (2018). Desafios na educação de profissionais de Saúde: uma abordagem interdisciplinar e interprofissional. Interface - (Botucatu), 22(supl.2), 1549-62. https://doi.org/10.1590/1807-57622017.0722

Lamers, J. M. S., \& Toassi, R. F. C. (2018). Perspectivas para a formação dos profissionais da saúde: educação interprofissional em foco. Revista saberes plurais: educação na saúde, 2(2), 34-42. https://seer.ufrgs.br/saberesplurais/article/view/75663/49184

Pereira, S.S., Costa, D. R. G. O., Camargo, C. A. C. M., Rosa, W. A. G., Graciano, C. S. M., \& Ribeiro, M. I. L. C. (2020). Pet health interprofissionality: dificulties pointed out by primary care teams in relation to mental health. Research, Society and Development, 9(7), e948975240-e948975240. https://doi.org/10.33448/rsd-v9i7.5240

Pereira, A. S., Shitsuka, D. M., Parreira, F. J., Shitsuka, R. (2018). Metodologia da Pesquisa Científica. https://repositorio.ufsm.br/bitstream/handle/1/1582 4/Lic_Computacao_Metodologia-Pesquisa-Cientifica.pdf?sequence=1

Reeves, S. (2016). Porque precisamos da educação interprofissional para um cuidado efetivo e seguro. Interface - Botucatu, 20(56), 185-196. https://doi.org/10.1590/1807-57622014.0092 
Research, Society and Development, v. 10, n. 1, e40610111862, 2021

(CC BY 4.0) | ISSN 2525-3409 | DOI: http://dx.doi.org/10.33448/rsd-v10i1.11862

Reeves, S., Xyrichis, A., \& Zwarenstein, M. (2018). Teamwork, collaboration, coordination, and networking: Why we need to distinguish between different types of interprofessional practice. Journal of Interprofessional Care, 32:1, 1-3. https://doi.org/10.1080/13561820.2017.1400150

Toassi, R. F. C. (2017). Interprofissionalidade e formação na saúde: onde estamos? Porto-Alegre: Rede Unida. http://historico.redeunida.org.br/editora/biblioteca-digital/serie-vivencias-em-educacao-na-saude/vol-06-interprofissionalidade-e-formacao-na-saude-pdf 